\title{
A Roadmap Focused on SMEs Decided to Participate in Collaborative Non-Hierarchical Networks
}

\author{
Beatriz Andrés and Raúl Poler \\ Research Centre on Production Management and Engineering (CIGIP), \\ Universitat Politècnica de València (UPV). Plaza Ferrándiz y Carbonell, 2, 03801 Alcoy, Spain \\ \{Beaanna, rpoler\}@cigip.upv.es
}

\begin{abstract}
The importance of collaboration has increased in supply networks; thus, the number of so called non-hierarchical manufacturing networks (NHN) has also increased. A roadmap to help SMEs to participate and create collaborative non-hierarchical networks is developed. Specifically, the methodology carried out in the NHNmap is described along the paper.
\end{abstract}

Keywords: roadmap, collaboration, non-hierarchical networks, SMEs.

\section{Introduction}

In recent years, researchers from different disciplines have shown a growing interest study in strengths of collaborative manufacturing networks along dynamic environments. As a result the concept of Non-Hierarchical Manufacturing Network (NHN) has emerged, changing from centralised (hierarchical network, HN) to decentralised decision making approaches. Collaborative participation requires greater exchanges of information, responsibilities sharing and partners' commitment as a whole. In collaborative NHN, SMEs are actively involved in network decisionmaking and problems are tackled together. NHN take into account the objectives of all the partners by equally considering all networked nodes [1].

Despite the growing interest posed in NHN, so far there is no roadmap that enables SMEs following common guidelines to participate or/and create collaborative NHN (section 2). In light of this, to carry out the roadmap, presented below, we have sought inspiration in a number of examples of roadmaps [2][3][4]. However, the proposed roadmap has developed its own and innovative procedure to help SMEs to participate in collaborative environments within a NHN (section 3).

\section{Roadmap Literature Overview}

According to [5] a roadmap is defined as the view of a group of how to get where they want to go or, achieve their desired objective and helps the group to ensure that the capacities to achieve their goals are in right place and time. Another definition is the roadmapping concept as the learning process and a communication tool for the 
group. [6] attempt to bring some common definition to roadmaps and roadmapping processes to unify the broad scope of roadmap objectives and uses.

Numerous gains are derived from developing roadmaps in collaborative contexts [6]: greater product flexibility, better quality, quickly solve technical problems, identify areas with high potential promise, improve collaboration of activities and resources in complex and uncertain environments, develop long-term sustainable relationships capable of adapting to changes in broader market demands, are few examples.

Various types of roadmaps, with rather different scopes and level of generality, can be considered [2]: (i) science and research, (ii) cross-industry, (iii) industry, (iv) technology, (v) product, (vi) product-technology and (vii) project and issue roadmaps.

Different frameworks and roadmaps have been developed in order to tackle problems when establishing collaborative relationships among networked SMEs.

First records of roadmapping application date back to 1980s at Motorola, to support technology planning to integrate markets, products and technologies [7]. Technology roadmaps became popular as an approach to strategic planning for the future of technology in different sectors [2]. A multidisciplinary approach involving the perspectives of technology is provided by [8] in order to deal with collaborationbased information technology solutions. In the same research line, [9] also propose a method of technology roadmapping.

Service orientation provides an architectural pattern able to cope with the needs of integrated and distributed collaborative solutions. [10] develop a roadmap into a major adoption of SOA to support agile reconfigurable supply chains. Furthermore, [11] propose a framework based on web service system to offer interoperability among distributed participants in a collaborative network $(\mathrm{CN})$, and their management information systems, and provides the appropriate support to all necessary decisionmaking steps towards the attainment of the network strategic common goals.

Interoperability problem in CNs is further addressed in the literature through roadmapping. A roadmap is proposed by [12] to deal with interoperability of enterprise applications and software. Another example is the roadmap developing the Unified Enterprise Modelling Language (UEML) as a result of the work of [13]. In order to allow the integration of systems and interoperability to operate in virtual enterprises, [14] design a strategic roadmap. Related with interoperability and sharing information, [15] present a preliminary version of a roadmap towards defining a standard to be used in a collaborative knowledge-based platform for information and knowledge sharing.

Furthermore, the innovation view in CNs is also addressed through roadmapping. In the research of inter-firm task partitioning, resource sharing activities and capability development behaviours, [16] contribute providing a roadmap in networked product innovation contexts. To deal with the implementation and evaluation for facilitating innovation in collaborative environments, [17] report another roadmap.

Roadmapping allows bringing together people from different parts of the $\mathrm{CN}$, giving an opportunity to share information and perspectives and providing a vehicle for holistic consideration of problems, opportunities and new ideas [18]. An example of a research agenda for CNs is given by the VOmap roadmap for advanced virtual organizations [2]. VOmap aims at identifying the key research challenges needed to 
fulfil the vision for the European initiative on dynamic collaborative VOs. Thus, the Reference Model for COllaborative Networks (ARCON) [19] is focused on a modelling approach considering multiple modelling perspectives: environment characteristics, life cycle and modelling in CNs. Furthermore, [20] propose a collaborative network approach to develop an integrated business service concept in VO context. With the purpose of identifying what component capabilities possess SMEs, what system capabilities are required and what skills must be developed to establish collaboration within a network, it is also proposed a roadmap [21]. A roadmapping initiative is introduced by [22] to address a strategic research plan covering the social, organizational, and technological perspectives in CNs. In order to jointly move towards integrated business solutions as well as a culture shift towards long-term trusted relationships, [23] provides the supply chain Integration Roadmap Organization developed both in-house and among supply chain partners. Continuing with the intra-/inter-organizational research, [24] address two kinds of behaviour, Individual Collaborative Behaviour and Network Collective Behaviour, and introduce an approach to compare networked partners in order to identify conflicts, select the best-fit collaborative members and assign roles and rights in CNs.

In order to properly design organizational structures, GloNet project matches SMEs needs and emerging models identified in the disciplines of collaborative networks [25]. Finally, [26] provide a method useful for any SME who does not have advanced knowledge of groupware and who needs collaborative modelling tools.

The literature review carried out enlighten that the developed roadmaps and frameworks deal with collaborative problems focused on technology, interoperability, services oriented, VO, modelling, information and knowledge sharing, innovation, measurement of collaboration, partners identification, alignment, collaborative behaviour, etc. Consequently, it is needed to develop a specific roadmap to the deal with the collaborative situation in NHN. Contrariwise the roadmaps and frameworks provided in the literature, the roadmap proposed in this paper provides a migration path between the current state of SMEs belonging to non-collaborative networks, and the long-term vision to achieve collaborative relationships in NHN, together with the linkages between layers, in a form that is flexible enough to be updated over time.

\section{NHNmap Vision}

When SMEs decide to participate in collaborative NHN, they have to deal with a series of problems arising from inter-organizational relationships. To do this, a generic roadmap is proposed, whatever the sector to which the NHN belong, in order to allow decision makers to identify problems concerning the establishment of collaboration in decentralised decision models, what characterises NHN.

The developed roadmap -NHNmap- identifies the weaknesses that arise in SMEs when decide to participate in collaborative NHN. Besides, NHNmap help SMEs to identify proper solutions to overcome the weaknesses derived from collaborative relationships characterised by decentralised decision making. To sum up, NHNmap 
provides the necessary research, in SMEs, to develop collaborative solutions for dealing with problems derived from NHN participation/formation.

The provided roadmap aims to (i) identify multiple perspectives and abstraction levels of collaborative problems, (ii) identify the problem design possibilities, (iii) analyse models, guidelines and tools (M/G/T) to cope with collaboration and (iv) design integrated and aligned solutions for collaborative NHN partners.

The NHNmap considers two important elements: the SMEs initial features when belonging to non-collaborative $N H N$ and the SME desired vision in collaborative NHN. Figure 1 represents the NHNmap goal, which develops a guide that enables SMEs to consider all transitional decisions necessary to achieve the desired future state of collaborative NHN.

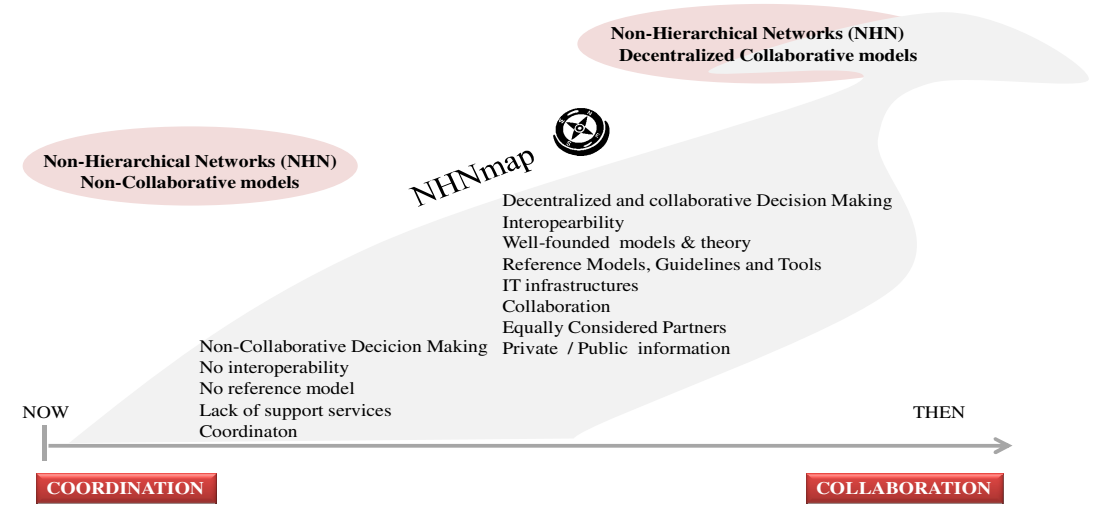

Fig. 1. NHNmap goal. Collaborative Non-Hierarchical Networks $\rightarrow$ TO BE and AS IS.

Collaborative NHN have been less studied due to the complexity of its structure. SMEs participation in collaborative NHN involves a change of environment, decision making and implementation processes regarding the traditional networks based on centralised decision systems. With the appearance of NHN it appears the need to lay the groundwork for new research on (i) collaborative NHN formation and (ii) SMEs needs when establishing collaborative relationships between partners belonging to a $\mathrm{NHN}$, in order to make more efficient networks.

Not all the problems arising from the collaborative processes among partners of the same network are fully considered in the proposed roadmap. However, problems that significantly influence in establishing collaboration between partners are contemplated to achieve the desired collaborative NHN.

Therefore, by considering the statements above, NHNmap vision is expressed as:

In the future, most companies will be part of collaborative networks characterised by decentralised decision making, such as NHN, and dynamic networks capable of rapid response to change market conditions. NHNmap will cover:

- Decentralised and collaborative decision making.

- Training and participation of partners in order to belong to collaborative NHN. 
- Interoperability in communications and collaborative infrastructures (IT/IS).

- Standardization and jointly business process.

- Distinction between private and public information.

- Well founded collaborative network models, guidelines and reference tools.

- Equally considered members.

- Management principles tailored to collaborative NHN behaviours.

- Collaboration mechanisms to deal with decentralized decision making.

Collaborative participation in NHN enables SMEs to create new opportunities, becoming more competitive and innovative, which implies greater risk-taking.

The contribution of the proposed roadmap is to allow SMEs to overcome the adaptation path for belonging to collaborative NHN.

NHNmap is structured from 4 focus areas: (i) collaboration establishment, (ii) proposition of $\mathrm{M} / \mathrm{G} / \mathrm{T}$ to overcome problems in collaborative processes, (iii) identification of IT/SI to support collaborative processes and decentralised decision models and (iv) performance evaluation of collaborative NHN participation.

Based on [5] many reasons to establish the NHNmap are provided below:

- NHNmap allows an orderly and planned evolution from non-collaborative NHN to collaborative NHN.

- $\quad$ NHNmap helps to establish objectives and strategies aligned with collaborative NHN partners.

- $\quad$ NHNmap provides a guide for NHN members, enabling partners to recognize and act on events that require changes of direction towards belonging to a collaborative NHN.

- NHNmap reveals gaps on SMEs capabilities when they want to take part in a collaborative NHN. Regarding to technology, decision making and culture.

- NHNmap allows identifying models, guidelines and tools to apply the proper solution in SMEs.

- NHNmap allows decision makers to agree and communicate research, technology and innovation plans to NHN collaborative members.

- $\quad$ NHNmap helps to identify priority investments in collaborative SMEs.

\section{$4 \quad$ NHNmap Phases}

Given the literature review carried out in section 2, no collaborative framework to identify the SMEs needs when deciding to participate in collaborative NHN has been already generated. In light of this, in this section the NHNmap is provided.

The developed roadmap helps SMEs, which have decided to participate in collaborative processes within a NHN, to start establishing decentralised collaborative relationships. NHNmap is multi-layered at network level and SMEs level (local level), reflecting the integration of technology, processes and collaborative perspectives among SMEs, including internal and external sources and supporting communication across partners and network boundaries. The adopted structure for 
defining the phases and sub-phases of the roadmap is important, reflecting fundamental aspects of the collaborative NHN and issues being considered.

Before implementing the NHNmap, all the processes that take place within the decentralised collaborative network must be identified for each SME.

NHNmap allow SMEs to align the businesses with collaborative networked partners and propose a set of common objectives, joint leadership, information sharing, processes adaptability to collaborative non-hierarchical networks, assessment and continuous reviewing results through a measurement system.

According to figure 2, the provided roadmap, NHNmap, consists of ten phases.

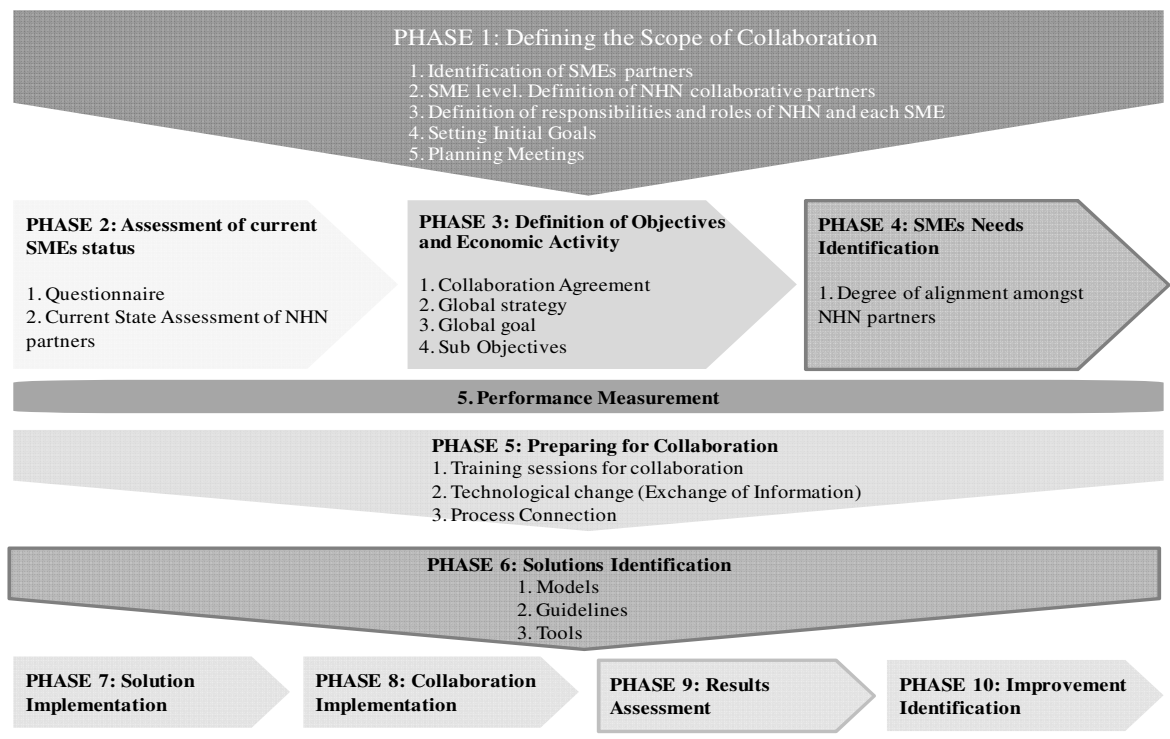

Fig. 2. NHNmap: roadmap to achieve collaborative non-hierarchical manufacturing networks

Each phase is briefly developed and explained next.

PHASE 1: Defining the scope of collaboration. The contact among SMEs, that decide to participate in collaborative NHN, is initiated. Furthermore, this phase allows networked partners to define the scope of collaboration. All NHN members are identified and defined in terms of the processes performed in the NHN: (i) procurement, (ii) production, (iii) distribution and (iv) marketing and sales. The degree of collaboration of each SME is also identified. After that, responsibilities, roles and initial objectives of each SME in the collaborative NHN are defined. A consultant manager can be designed in order to monitoring the NHNmap sequence. Phase 1 allows collaborative partners establishing meetings in each roadmap phase to collect information, undertake further stages and collect feedbacks from each partner.

PHASE 2: Assessment of Current SMEs Status. The current status of each SME, willing to participate in collaborative $\mathrm{NHN}$, is evaluated. Before starting the activity in the collaborative NHN, networked partners assess their business needs, values, 
culture, strategies and trading partner relationships in order to implement best practices. After this phase, networked SMEs exactly know whether they are ready to link its activities in a collaborative network characterised by decentralised decision making. Thus, a questionnaire must be filled for identifying SME areas that need immediate attention, in order to participate in a collaborative NHN. Results achieved in phase 2 helps NHN partners to recognize their status and identify where change is needed to successfully implement collaborative partnerships in NHN.

PHASE 3: Definition of Objectives and Economic Activity. The objectives and economic activity, to carry out in the collaborative NHN, are defined. At the end of this phase, SMEs are able to define the objectives and the economic activity to perform within the collaborative NHN. The collaboration agreement is firstly defined to enable collaborative nodes following instructions and rules for governing the relationship among NHN members. After defining the collaboration agreement the global strategy is defined; then, the global objective is determined to describe the purpose of economic activity. The global objective consists of different sub-objectives that have to be achieved by SMEs in order to reach the activity at the defined network level. For better collaborative network participation, the performance measurement system (PMS) is designed. The elements of the performance measurement must be based on the objectives, strategies, critical success factors and key performance indicators (KPIs) at different levels of the network.

PHASE 4: SMEs Needs Identification. A methodology to identify the needs and weaknesses that arise in SMEs willing to collaborate in a collaborative NHN is provided. The methodology consists of an analysis that identifies the SMEs needs and associates them with a list of problems through a 4-dimensional analysis: (i) strategy, (ii) technology, (iii) membership and (iv) product. The given methodology identifies the weaknesses for establishing collaborative relationships with partners belonging to a collaborative NHN.

PHASE 5: Preparing for Collaboration. Networked partners must be able to work in an integrated way, while individually must maintain its independence and autonomy. This phase identifies the technology and additional resources required to support collaboration. Collaborative NHN are known for establishing decentralised decision making models, and NHN members must define rules for collaboration to cope with the dynamism that characterizes the network environment. Phase 5 provides training sessions to collaborate, and identifies areas that need further attention in the development of behaviour patterns. Furthermore, in this phase the technological change of view is overcome as a critical factor. Prior to technological change, there should be reviewed existing technologies and skills that organizations possess for helping partners to communicate and collaborate, taking into account the principles of interoperability (phase 3). Technological change is directly involved with information exchange. The exchange of information between companies in the same NHN is done taking into account the following aspects: (i) technology infrastructure and platforms (ii) federated information management (iii) visibility and access rights (iv) standards (v) data logging and (vi) data synchronization. Besides, process connection must be 
also considered through (i) process reengineering (ii) collaborative transactions and (iii) distributed platforms.

PHASE 6: Solutions Identification. This phase identifies solutions to overcome SMEs needs, previously identified in Phase 4. Furthermore, allows NHN partners identifying solutions to deal with concerns expressed at the phase of preparation for collaboration (Phase 5). In this phase problems arising from collaboration between SMEs when participating in collaborative networks are analysed. Those problems are the result from inter-organizational barriers such as lack of confidence, lack of knowledge exchange and flow, lack of leadership and lack of change vision. Definitely, problems and associated supporting solutions are collected and structured, determining the extent to which these solutions can be adopted to address problems in collaborative NHN. Provided solutions are classified into models, guidelines and tools (Figure 3).

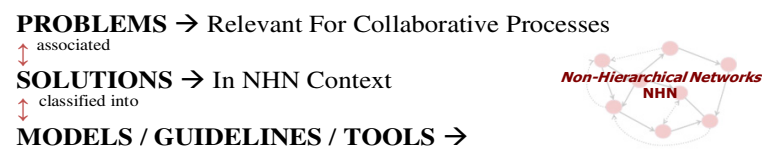

Fig. 3. Solutions identification

PHASE 7: Solution Implementation. A guideline to implement the solutions identified in phase 6 is provided. This step permits to identify the extent to which the considered solutions can be applied in the SMEs, taking into account the capabilities and resources they have.

PHASE 8: Collaboration Implementation. Collaboration within NHN partners is started in this phase. NHN members begin to collaborate and exchange information with each other. Networked partners adopt solutions to meet with collaborative conditions. In this phase, personal in charge for executing and implementing technology increase their experience in the ICT arena. Collaborative partners start to establish collaborative process and technologies. After a period of time the NHN management team meets to discuss the progress, problems and changes.

PHASE 9: Results Assessment. In this evaluation phase, NHN managers review the progress results. SMEs managers report the assessment results obtained from the collaborative participation in the NHN. Data recorded from the evaluation allow NHN managers to identify and generate performance improvements for SMEs (phase 10). The loop of performance measurement (phase 4) and evaluation (phase 9) will be periodically repeated. Furthermore, it is recommended to agree the review period before implementing NHN collaborative initiatives. This phase is specifically relevant to assess the collaboration results, and provides an excellent opportunity to report on the progress of collaborative NHN formation and participation.

PHASE 10: Improvement Identification. Future lines of action in the collaborative NHN are addressed in this stage. NHN partners must identify and decide further actions, carried out by SMEs, relating to collaboration. This phase allows decision makers to orderly capture future justified SMEs initiatives to achieve the desired 
collaborative environment. This stage is developed taking into account the evaluated results obtained in phase 9. Efforts to determine further SMEs actions can be aimed at improving collaborative relations either within the same NHN or forming a second NHN with different partners.

A joint report to communicate all partners the NHN collaborative decisions agreed in the collaborative process should be published at the network level. The report must give shape a common project and the potential impact of collaboration. SMEs managers are responsible of elaborating this report to record the assessment of results and further actions that must be developed to efficiently participate in collaborative relationships within NHN. NHN managers review the results of progress as regards evaluation results. The main aim of recording the evaluation results in phase 10 is to identify and apply improvements in SMEs when belonging to collaborative NHN.

\section{Conclusions}

Inter-enterprise collaboration in NHN allows SMEs to increase competitiveness, agility and dynamicity in today's global market. Empowering SMEs to establish collaborative relationships, within the network they belong, is a key question for reinforcing the necessary conditions to participate in collaborative NHN. In terms of research, collaborative NHN involve a significant activity due to the reached benefits both in network and SMEs level. Amongst problems related with NHN we find specifically interesting the initiative related with the evolution from non-collaborative NHN to collaborative ones. This paper focuses on supporting the path to be carried out by SMEs decided to participate in a collaborative NHN. As a result, it is required to start a research in collaborative NHN context. NHNmap provides a strategic tool focused on creating the necessary mechanisms for establishing collaborative NHN.

Research work, in collaborative NHN, has been launched only few years ago. That is to say, the most of the work has to be done in the next future, and we are in the early stage of development: SMEs as-is analysis, requirements definition, roadmaps, case studies, etc. Therefore, NHNmap is a dynamic construct that needs to be periodically revised its implementation, taking into account new trends.

Future research is aimed at implement the NHNmap in different organizations and sectors. This will be fundamental to complete the external validation of the roadmap in order to demonstrate the NHNmap potential benefits. The original contribution of this work is that NHNmap is developed at both network and SMEs levels and serves as a beginning of application to create collaborative NHN.

\section{References}

1. Intelligent Non-Hierarchical Manufacturing Networks (iNet-IMS) (February 2012), http://www.ims.org/sites/default/files/iNetIMS 20 MTP $\% 20$ Initiative $202009 \% 20 \mathrm{v} 1.3$. doc 
2. Camarinha-Matos, L., Afsarmanesh, H.: A RoadMap for strategic research on Virtual Organizations. In: Camarinha-Matos, L.H., Afsarmanesh, H. (eds.) Proceedings of the 4th IFIP Working Conference on Infrastructures for Virtual Enterprises (PRO-VE 2003). Kluwer Academic Publishers (2003)

3. VICS, Voluntary Interindustry Commerce Standards (2012),

http: / / www.vics.org/committees/cpfr/,

http://www.vics.org/docs/guidelines/cpfr_roadmap_case_

studies/06_3_Roadmap_To_CPFR.pdf

4. NZBCSD -New Zealand Business Council for Sustainable Development (2003), http: / /www.nzbcsd.org.nz / supplychain/

5. Albright, R.E.: A roadmapping perspective: Science-driven technologies (2002), http://www.albrightstrategy.com/papers/A_Roadmapping_ Perspective-Albright-09-26-02.pdf

6. Kostoff, R.N., Schaller, R.R.: Science and Technology Roadmaps. IEEE Transactions on Engineering Management 48(2), 132-143 (2001)

7. Rinne, M.: Technology roadmaps: Infrastructure for innovation. Technological Forecasting \& Social Change 71(1/2), 67-80 (2004)

8. Osório, A.L., Camarinha-Matos, L.M., Afsarmanesh, H.: Cooperation Enabled Systems for Collaborative Networks. In: Camarinha-Matos, L.M., Pereira-Klen, A., Afsarmanesh, H. (eds.) PRO-VE 2011. IFIP AICT, vol. 362, pp. 400-409. Springer, Heidelberg (2011a)

9. Caetano, M., Amaral, D.C.: Roadmapping for technology push and partnership: A contribution for open innovation environments. Technovation 31, 320-335 (2011)

10. Cândido, G., Barata, J., Colombo, A.W., Jammes, F.: SOA in reconfigurable supply chains: A research roadmap. Engineering Applications of Artificial Intelligence 22, 939949 (2009)

11. Lin, H.W., Nagalingam, S.V., Kuik, S.S., Murata, T.: Design of a Global Decision Support System for a manufacturing SME: Towards participating in Collaborative Manufacturing. Int. J. Production Economics 136, 1-12 (2012)

12. Chen, D., Doumeingts, G.: European initiatives to develop interoperability of enterprise applications-basic concepts, framework and roadmap. Annual Reviews in Control 27, 153$162(2003)$

13. Ducq, Y., Chen, D., Vallespir, B.: Interoperability in enterprise modelling: requirements and roadmap. Advanced Engineering Informatics 18, 193-203 (2004)

14. Rezgui, Y., Zarli, A.: Paving the Way to the Vision of Digital Construction: A Strategic Roadmap. J. of Construction Engineering and Management, 767-776 (2006)

15. Azouzi, R., d'Amours, S.: Standards for information and knowledge sharing in collaborative design of planning systems within the forest product industry. CILRET 46 (2011)

16. Perks, H., Moxey, S.: Market-facing innovation networks: How lead firms partition tasks, share resources and develop capabilities. Industrial Marketing Management 40, 1224-1237 (2011)

17. Budweg, S., Schaffers, H., Rulanda, R., Kristensenc, K., Prinz, W.: Enhancing collaboration in communities of professionals using a Living Lab approach. Production Planning \& Control 22(5-6), 594-609 (2011)

18. Phaal, R., Farrukh, C.J.P., Probert, D.R.: Technology roadmapping - A planning framework for evolution and revolution. Technological Forecasting \& Social Change 71, 5-26 (2004)

19. Camarinha-Matos, L.M., Afsarmanesh, H.: On reference models for collaborative networked organizations. Int. J. of Production Research 46(9), 2453-2469 (2008) 
20. Osório, L., Afsarmanesh, H., Camarinha-Matos, L.M.: A Service Integration Platform for Collaborative Networks. Studies in Inf. and Control 20(1), 19-30 (2011b)

21. Barton, R., Thomas, A.: Implementation of intelligent systems, enabling integration of SMEs to high-value supply chain networks. Engineering Applications of Artificial Intelligence 22, 929-938 (2009)

22. Camarinha-Matos, L.M., Afsarmanesh, H.: Active Ageing Roadmap - A Collaborative Networks Contribution to Demographic Sustainability. In: Camarinha-Matos, L.M., Boucher, X., Afsarmanesh, H. (eds.) PRO-VE 2010. IFIP AICT, vol. 336, pp. 46-59. Springer, Heidelberg (2010)

23. Hvolby, H.H., Trienekens, J.H.: Challenges in business systems integration. Computers in Industry $61,808-812$ (2010)

24. Shadi, M., Afsarmanesh, H.: Addressing Behavior in Collaborative Networks. In: Camarinha-Matos, L.M., Pereira-Klen, A., Afsarmanesh, H. (eds.) PRO-VE 2011. IFIP AICT, vol. 362, pp. 263-270. Springer, Heidelberg (2011)

25. Camarinha-Matos, L.M., Afsarmanesh, H., Koelmel, B.: Collaborative Networks in Support of Service-Enhanced Products. In: Camarinha-Matos, L.M., Pereira-Klen, A., Afsarmanesh, H. (eds.) PRO-VE 2011. IFIP AICT, vol. 362, pp. 95-104. Springer, Heidelberg (2011)

26. Gallardo, J., Bravo, C., Redondo, M.A.: A model-driven development method for collaborative modeling tools. J. of Network and Computer Applications 35, 1086-1105 (2012) 